\title{
Traveler's Infections: Understanding SARS-CoV-2 as a Potential Agent
}

\author{
Victor Baba Oti ${ }^{*}$, Marina Ioannou ${ }^{2}$
}

\author{
${ }^{1}$ Department of Microbiology, Nasarawa State University, Keffi, Nigeria \\ ${ }^{2}$ School of Life Sciences, University of Essex, Cholchester, the United Kingdom
}

\begin{abstract}
Coronavirus disease 2019 (COVID-19) has become a major, global, public health challenge. Over 1,051,635 confirmed cases have been reported worldwide, and both local and international travelers are considered to represent populations at high risk of acquiring the infection. Many factors are involved in the spread of this viral agent, including travel to and from endemic areas, a history of contact with a traveler from an endemic country, the virus infection rate at the destination, individual health, and inadequate prevention and control measures. The virus is thought to be transmitted through respiratory droplets, generated by coughing or sneezing and spread through close contact with infected persons or contaminated surfaces. Diagnostic strategies include detection of antiviral antibodies, multiplex nucleic acid amplification, and microarray-based assays. Currently, no approved antiviral therapy or vaccine exists to treat or prevent the infection. Public health measures have been enforced, to slow spread of the virus, by governments. The World Health Organization (WHO) have recommended screening be performed at airports, for all travelers, before traveling to endemic countries and the restriction of travel to countries more than one thousand cases of infection. In response to the global outbreak, this review examines COVID-19 as a potential traveler's infection.
\end{abstract}

Keywords: COVID-19, incidence rate ratio, social restriction, time series analysis

\section{Introduction}

Severe acute respiratory syndrome coronavirus 2 (SARS-CoV-2) is an enveloped, single-stranded, positivesense, ribonucleic acid (RNA) virus that causes coronavirus disease 2019 (COVID-19), a zoonotic infectious disease that has been declared a global, public health emergency of international concern (PHEIC).1,2 The infection was reported to originate from Wuhan, Hubei Province, China, in 2019, and has been linked to Wuhan's Huanan Seafood Wholesale Market. Bats are reportedly the major natural reservoir of the virus, and a 5-year study indicated the coexistence of highly diverse SARS-CoVs in bat populations found in caves in Yunnan Province, China. ${ }^{3}$ A recombination event may have facilitated the 'spillover' from the virus's original host to a new host, humans. The viral agent may have been disseminated, from bats to an unknown intermediate host, followed by the infection of human beings. ${ }^{4,5}$ The viral infection has since spread to virtually all parts of the globe, due to travel and the global migration of infected and exposed individuals. ${ }^{6}$ On March 11, 2020, the World Health Organization (WHO) declared COVID-
19 to be a pandemic, due to its spread to almost all six WHO regions. ${ }^{7}$ A reported 1,051,635 (and counting) confirmed cases have been identified, worldwide, and both local and international travelers represent populations at high risk of acquiring the infection. ${ }^{7,8}$ Coronaviruses usually cause mild respiratory infections; however, the symptoms of SARS-CoV-2 infections can range from mild, including fever, cough, and difficulty breathing, to severe, causing pneumonia and multi-organ failure. Elderly individuals and individuals with pre-existing medical conditions are at high risk of progressing speedily and swiftly into acute respiratory distress syndrome, septic shock, and coagulation dysfunction, which can result in death. ${ }^{1}$ This review examines SARS-CoV-2 infections and COVID-19 as a potential traveler's disease.

\section{The Structure of Severe Acute Respiratory Syndrome Coronavirus 2 (SARS-CoV-2)}

SARS-CoV-2 also known as 2019-nCoV,2 is an enveloped, single-stranded, positive-sense RNA virus, with a 3'-poly-A tail and a 5'-cap structure. The name corona- 
virus derives from the crown-like characteristic appearance under electron microscopy (corona is the Latin term for crown), due to the presence of spike glycoproteins on the envelope 9 (Figure1).

SARS-CoV-2 is a strain of SARS-related coronaviruses, from the taxonomic perspective. SARS-CoV-2 belongs to the family Coronaviridae, in the genus Betacoronavirus. ${ }^{11}$ The genetic material found in SARS$\mathrm{CoV}-2$ is approximately $30 \mathrm{~kb}$ in length, which makes it the largest known genome that has been identified in an RNA virus. The SARS-CoV-2 virus is one of seven Coronaviridae capable of infecting human beings, in addition to NL63, HKU1, 229E, OC43, MERS-CoV, and SARS-CoV.12,13 The genome of a typical coronavirus contains a minimum of six open-reading frames (ORFs). Approximately $70 \%$ of the viral genome encodes the polyprotein pp1ab, which is further cleaved into 16 nonstructural proteins, which play roles in genome replication and transcription through the viral-encoded chymotrypsin-like protease (3CLpro), in synergy with one or two papain-like proteases. ${ }^{14,15}$ The other $30 \%$ of the genome encodes four structural proteins, including spike $(\mathrm{S})$, envelope (E), membrane $(\mathrm{M})$, and nucleocapsid $(\mathrm{N})$, and additional helper proteins. The $\mathrm{S}$ protein plays a significant role, mediating membrane fusion and receptor binding, which, in turn, determines the host tropism of the virus. ${ }^{16,17}$

\section{Transmission Routes and Diagnosis of COVID-19}

Epidemiological studies in Wuhan were the first outbreak was reported, associated the virus with a sea food market where live animals were sold, with many patients having worked in, or visited the market. ${ }^{4,5}$ Subsequently, secondary cases begun to appear approximately ten days later. ${ }^{18}$ Although the new patients had no direct contact with the seafood market, contact tracing showed that they had contacts with inhabitants near the market. ${ }^{19}$ Advanced genetic and virologic studies have found that bats represent natural reservoirs of this viral agent. ${ }^{4,20}$ Studies have demonstrated that most bat CoVs have similar genomes as those found in beta-CoVs and alphaCoVs. ${ }^{19,21}$

The virus can be identified through different laboratory methods, including the rapid testing of Immunoglobulin $\mathrm{G}(\operatorname{IgG})$ and Immunoglobulin $\mathrm{M}(\mathrm{IgM})$ levels, electron microscopy, cell-culturing, and nucleic acid detection reverse transcriptase-polymerase chain reaction (RT-PCR), which is currently the gold standard for SARS-CoV-2 testing and is performed on a nasopharyngeal sample. When an individual is confirmed to be infected, contact tracing is performed, during which a detailed history of all persons in contact with a confirmed patient is examined, using precise laboratory testing. 1,22 SARS-CoV-2 infections can also be determined by a myr-

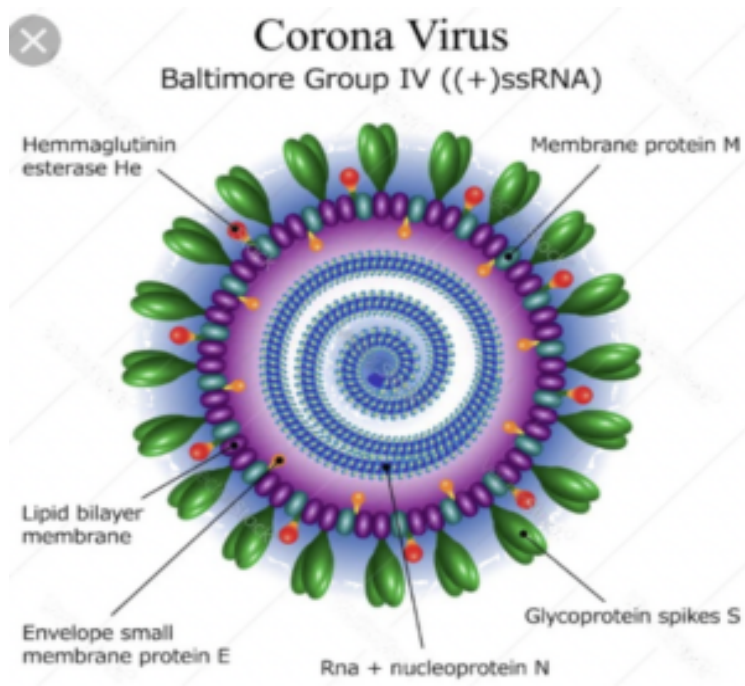

Figure 1. SARS-CoV-2 Morphology with the Minimal Set of Structural Proteins 9-10

iad of clinical features, predictors, a chest computed tomography (CT) scans showing pneumonia characteristics and others. ${ }^{23,24}$ As of March 19, 2020, no antibodybased techniques have been developed, although serious ongoing studies are attempting to develop these techniques. ${ }^{22}$

\section{Clinical Features of SARS-CoV-2 Infection}

The viral agent gains entry into the respiratory tract and infects the cells of the respiratory system, 25,26 causing acute respiratory disease, with a high prevalence among travelers. ${ }^{27}$ The incubation period for the viral infection is approximately $2-14$ days, with an average incubation period of 5 days. ${ }^{19}$ The infection is usually asymptomatic among those with a travel history to endemic countries, but can also present with symptoms similar to those observed during flu infections, including fever, dry cough, and difficulty breathing. ${ }^{14,28}$ When these symptoms are present, the difficulty breathing and associated chest pain and pressure persists, resulting in walking difficulties, confusion, and a bluish tint to the lips and face. These symptoms indicate a critical situation that requires immediate medical attention. ${ }^{14,29}$ Zhang, et al.,22 reported the presence of the virus in fecal and blood samples, suggesting the possibility of multiple transmission routes. The identification of the viral receptor, angiotensin-converting enzyme 2 (ACE2), which is often present in large quantities on enterocytes in the small intestines and epithelial cells in the lung alveoli, is likely to assist researchers in understanding infection routes and the pathogenesis of the viral agent. ${ }^{30}$ 


\section{Aspects of COVID-19 Epidemiology as a Traveler's Infection}

In December 31, 2019, the novel SARS-CoV-2 outbreak was first reported in Wuhan City, Hubei Province, China, ${ }^{31}$ as pneumonia outbreak without a known causal agent. The WHO announced that the etiology was a new coronavirus, which was temporarily referred to as2019$\mathrm{nCoV}$, and was posited to be the causal agent for the infection on January $12,2020 .{ }^{32}$ Similar to SARS, the COVID-19 outbreak is thought to have initiated at a local seafood market, the Huanan Seafood Wholesale Market, and $2 / 3$ of the first 41 confirmed cases were linked to the market. Within a few weeks, the virus had spread to other countries, including those in Europe. Most SARSCoV-2 cases reported during the first 6 days (January 2328,2020 ) were associated with direct contact with someone who traveled to or from Wuhan. ${ }^{33}$ In Germany, a 33-year-old man (patient 1) became ill with cold and flu symptoms, on January 24, 2020, after attending a meeting in Munich with a Chinese business partner, on January 20, 2020.21 The business partner was visiting from Shanghai and she did not experience any signs or symptoms of illness. In Italy, a Bayesian phylogenetic reconstruction suggested that the virus found in Italy was imported by Chinese travelers, who were infected before they arrived in Italy. ${ }^{34}$ Japan identified its first case of viral infection on January 16, 2020, associated with a Chinese traveler from Wuhan. ${ }^{35}$ Australia's first case was identified on January 25, 2020, associated from a man who traveled to Wuhan. ${ }^{36}$ On January 20, 2020, the USA identified its index case, a traveler who returned from Wuhan, China. ${ }^{37}$ On January 24, 2020, France identified its first confirmed case of the novel coronavirus, which was also connected with a travel history to China. ${ }^{38}$ A traveler from Wuhan and Qom imported the first case of the infection from China to Iran on January 19, 2020. ${ }^{39}$ The index case for Pakistan was identified on February 26,2020 , when a young man with travel history to Karachi tested positive for the virus. ${ }^{40}$ On March 10, 2020, Turkey reported its index case, in a Turkish male who traveled to Europe. ${ }^{41}$ The initial report of viral infection in South Africa was March 5, 2020, in a patient who had traveled to Italy with his wife. ${ }^{42}$ The WHO has reported that the median age of people testing positive for the virus was approximately 45 years and that over two-thirds of those infected were males. ${ }^{18}$ The mortality rate among confirmed positive cases was $4.5 \%$, as of March 25, 2020, and appears to range from $0.2 \%-15 \%$, depending on age group and other health-related factors. 18 The fatality rates differ among countries and over time, based on differences in the rate of diagnosis and due to differences in access to adequate healthcare workers and facilities.

\section{Prevention and Control of SARS-CoV-2 Infection}

Preventive measures that have been implemented against the spread of the novel coronavirus include staying at home, practicing social distancing (avoiding crowded places), washing hands with soap and running water, regularly, for at least 20 seconds, good personal hygiene practices and the avoidance of picking or touching the nose, eyes, mouth, and other parts of the face with unwashed hands. ${ }^{43-45}$ Recommendations by the Centers for Disease Control and Prevention (CDC) included covering the nose and mouth with tissues when sneezing or coughing or using the inside of the elbow when tissue availability is scarce. ${ }^{46}$ Social distancing, which refers to people remaining at least 6 feet apart from one another, was introduced to reduce contact between people carrying the virus and uninfected populations. Workplaces and schools were closed, travel restrictions were implemented, and mass gatherings of more than 50 individuals were canceled. ${ }^{47}$ According to the $\mathrm{WHO}$ and CDC, surgical masks should only be used when an individual is symptomatic or is administering care to infected or suspected-infected persons, such as health workers. 43,44

Measures that benefit the community, such as the cancellation of mass gatherings, social distancing, the closing of schools, and the creation of plans that allow employees to work from home can help mitigate the dissemination of SARS-CoV-2 infections, and these types of preventive measures have been recommended by different CDC agencies, worldwide. ${ }^{46,47}$ Public health experts should provide travelers with information and guidelines regarding how they can mitigate their overall risks of viral infections and other respiratory complications, which can be disseminated through travel agencies, conveyance operators, travel health clinics, and at points of entry. 46

\section{Possible Treatments and Vaccine Candidates for COVID-19 Infection}

A variety of treatment methods and antiviral agents are being explored for the potential treatment of SARSCoV-2 infections, and some are progressing to the clinical trial stage. ${ }^{48}$ WHO has launched "Solidarity", which is a multi-country trial including 10 countries, in response to the COVID-19 pandemic, on March 2020.44 The following drugs are being studied: chloroquine and hydroxychloroquine, ritonavir / lopinavir and remdesivir in synergy with beta interferon. ${ }^{45,49}$ Scientific reports exist showing that remdesivir can be used to treat the viral infection, ${ }^{50}$ because it inhibits SARS-CoV-2 RNA transcription, in vitro.

Chloroquine, a drug that is currently used to treat malaria, is another potential drug that is currently being tested in China, with positive preliminary evidence. ${ }^{4,51}$ Chloroquine and hydroxychloroquine can effectively stop 
the effects of the viral agent, in vitro, and hydroxychloroquine has been shown to be more potent than chloroquine, with a more tolerable safety profile. ${ }^{47}$ Chloroquine and hydroxychloroquine have been listed as drugs with good preliminary outcomes for COVID-19 therapy, by the Italian Pharmaceutical Agency (IPA) in March 17, 2020.46,52

Currently, no vaccines are available for SARS-CoV-2 infections; however, vaccine development is crucial for inducing herd immunity and the possible eradication of this disease. ${ }^{43}$ Various ongoing studies are examining mRNA, epitope, and S protein-RBD structure-based vaccine candidates. Models of the human ACE2 protein in transgenic mice and rhesus monkeys have been wellmapped for the creation of a vaccine, and some SARS$\mathrm{CoV}-2$ vaccines candidates are already entering the clinical trial stage. ${ }^{53}$ In Seattle, USA, the first human trials for a vaccine candidate began on March 16, 2020, with just four volunteers. ${ }^{54}$ The vaccine candidate contains a harmless genetic code, copied from the SARS-CoV-2 virus that causes disease. Recombinant interleukin-7 (IL7) therapy is also being investigated, as this protein plays a significant function in the reproduction and maturation of lymphoid cells. ${ }^{55}$

\section{Conclusion}

Global migration, changes in climate, and evolving human-animal interactions are possible factors that may enhance the COVID-19 pandemic. Travelers are at high risk of acquiring the infection, and most countries with high numbers of cases have placed travel bans on foreign travel. We have provided a summarized review of the COVID-19 pandemic as a potential agent for traveler's infections, worldwide.

\begin{abstract}
Abbreviations
COVID-19: Coronavirus disease 2019; SARS-CoV-2: Severe Acute Respiratory Syndrome Coronavirus 2; RNA: Ribonucleic Acid; PHEIC: Public Health Emergency of International Concern; WHO: World Health Organization; ORFs: Open-reading Frames; S: Spike; E: Envelope; M: Membrane; N: Nucleocapsid; IgG: Immunoglobulin G; IgM: Immunoglobulin M; RT-PCR: Reverse Transcriptase-Polymerase Chain Reaction; ACE2: Angiotensin-Converting Enzyme 2; CDC: Centers for Disease Control and Prevention.
\end{abstract}

\section{Ethics Approval and Consent to Participate}

Not Applicable

\section{Competing Interest}

Authors declares that there is no significant competing financial, professional, or personal interests that might have affected the performance or presentation of the work described in this manuscript.

\section{Availability of Data and Materials}

The datasets used and/or analysed during the current study are available from the corresponding author on reasonable request.

\section{Authors' Contribution}

Victor B. Oti conceptualized and designed the study. Victor B. Oti and Marina Ioannou searched the literature and prepared the manuscript. All authors reviewed the manuscript and approved the final manuscript.

\section{Acknowledgment}

We appreciate the financial support of Mrs. Augustina C. Oti. The authors dedicate this review to the blessed memory of P. B. Oti, PhD and those who lost their lives due to COVID-19.

\section{References}

1. Adhikari SP, Meng S, Wu Y, Mao Y, Ye R, Wang Q, et al. Epidemiology, causes, clinical manifestation and diagnosis, prevention and control of coronavirus disease (COVID-19) during the early outbreak period: a scoping review. Infectious Diseases of Poverty. 2020; 9: 29.

2. World Health Organization. Emergencies preparedness, response: novel coronavirus - China; January 12, 2020.

3. Cui J, Li F, Shi ZL. Origin and evolution of pathogenic coronaviruses. Nature Reviews Microbiology. 2019; 17: 181-92.

4. Lu R, Zhao X, Li J, Niu P, Yang B, Wu H, et al. Genomic characterisation and epidemiology of 2019 novel coronavirus: implications for virus origins and receptor binding. The Lancet. 2020; 395: 565-74.

5. Zhou P, Yang XL, Wang XG, Hu B, Zhang L, Zhang W, et al. A pneumonia outbreak associated with a new coronavirus of probable bat origin. Nature. 2020; 579: 270-3.

6. Jin Y, Yang H, Ji W, Wu W, Chen S, Zhang W, et al. Virology, epidemiology, pathogenesis, and control of COVID-19. Viruses. 2020; 12 (4): 372.

7. World Health Organization. Novel coronavirus (2019- nCoV): situation Report-22; February 11, 2020.

8. Rothan HA and Byrareddy SN. The epidemiology and pathogenesis of coronavirus disease (COVID-19). Journal of Autoimmunity. 2020; 109: 102433.

9. Wrapp D, Wang N, Corbett KS, Goldsmith JA, Hsieh CL, Abiona O, et al. Cryo-EM structure of the 2019-nCoV spike in the prefusion conformation. Science. 2020; 367 (6483): 1260-63.

10. Tang X, Wu C, Li X, Song Y, Yao X, Wu X, et al. On the origin and continuing evolution of SARS-CoV-2. National Science Review. 2020; 7 (6): 1012-23.

11. Letko M, Marzi A, Munster V. Functional assessment of cell entry and receptor usage for SARS-CoV-2 and other lineage B betacoronaviruses. Nature Microbiology. 2020; 5: 562-9.

12. Wu F, Zhao S, Yu B, Chen Y M, Wang W, Hu Y, et al. Complete genome characterisation of a novel coronavirus associated with severe human respiratory disease in Wuhan, China. bioRxiv; 2020.

13. Lai CC, Shih TP, Ko WC, Tang HJ, Hsueh PR. Severe acute respiratory syndrome coronavirus 2 (SARS-CoV-2) and coronavirus disease2019 (COVID-19): the epidemic and the challenges. International Journal of Antimicrobial Agents. 2020; 55 (3): 105924. 
14. Chen N, Zhou M, Dong X, Qu J, Gong F, Han Y, et al. Epidemiological and clinical characteristics of 99 cases of 2019 novel coronavirus pneumonia in Wuhan, China: a descriptive study. The Lancet. 2020; 395: 507-13.

15. Xu H, Zhong L, Deng J, Peng J, Dan H, Zeng X, et al. High expression of ACE2 receptor of 2019-nCoV on the epithelial cells of oral mucosa. International Journal of Oral Science. 2020; 12: 8.

16. Lu G, Wang Q, Gao GF. Bat-to-human: spike features determining 'host jump' of coronaviruses SARS-CoV, MERS-CoV, and beyond. Trends in Microbiology. 2015; 23 (8): 468-78.

17. Wang Q, van der Velde R, Su Z, Wen J. Aquarius L-band scatterometer and radiometer observations over a Tibetan Plateau site. International Journal of Applied Earth Observation and Geoinformation. 2016; 45 Part B:165-77.

18. World Health Organization. Novel coronavirus (2019-nCoV): situation report-11; 2020.

19. Sahin AR, Erdogan A, MutluAgaoglu P, Dineri Y, Cakirci AY, Senel ME, et al. 2019 novel coronavirus (COVID-19) outbreak: a review of the current literature. Eurasian Journal of Medicine and Oncology. 2020; 4 (1): 1-7.

20. Zhang H, Penninger JM, Li Y, Zhong N, Slutsky AS. Angiotensin-converting enzyme 2 (ACE2) as a SARS-CoV-2 receptor: molecular mechanisms and potential therapeutic target. Intensive Care Medicine. 2020; 46: 586-90.

21. Rothe C, Schunk M, Sothmann P, Bretzel G, Froeschl G, Wallrauch C, et al. Transmission of 2019-nCoV Infection from an Asymptomatic Contact in Germany. New England Journal of Medicine. 2020; 382: 970-1.

22. Zhang W, Du RH, Li B, Zheng XS, Yang XL, Hu B, et al. Molecular and serological investigation of 2019-nCoV infected patients: implication of multiple shedding routes. Emerging Microbes \& Infections. 2020; 9 (1): 386-9.

23. Lei J, Li J, Li X, Qi X. CT imaging of the 2019 novel coronavirus (2019-nCoV) pneumonia. Radiology. 2020; 295: 18.

24. Corman VM, Landt O, Kaiser M, Molenkamp R, Meijer A, Chu DKW, et al. Detection of 2019 novel coronavirus (2019-nCoV) by real-time RT-PCR. Eurosurveillance. 2020; 25 (3).

25. Huang C, Wang Y, Li X, Ren L, Zhou J, Hu Y et al. Clinical features of patients infected with 2019 novel coronavirus in Wuhan, China. The Lancet. 2020; 395 (10223): 497-506.

26. Gralinski LE, Menachery VD. Return of the coronavirus: 2019-nCoV. Viruses. 2020; 12 (2): 135.

27. Hoehl S, Bojkova D, Berger A, Kortenbusch M, Cinatl J, Bojkova D, et al. Evidence of SARS-CoV-2 infection in returning travelers from Wuhan, China. New English Journal of Medicine. 2020; 382: 1278-80.

28. Centre for Disease Control and Prevention. Coronavirus Disease 2019 (COVID-19): symptoms; February 10, 2020 [Archived from the original on January 30, 2020].

29. Zhu N, Zhang D, Wang W.et al. A novel coronavirus from patients with pneumonia in China, 2019. New England Journal of Medicine. 2020; 382: 727-33.

30. Du Toit A. Outbreak of a novel coronavirus, Nature Reviews Microbiology. 2020; 18: 123.

31. World Health Organization. Novel Coronavirus (2019-nCoV): situa- tion report-40; 2020.

32. World Health Organization. 2019-nCoV: situation report-24; 2020.

33. Fan J, Liu X, Pan W, Douglas MW, Bao S. Epidemiology of 2019 novel coronavirus disease-19 in Gansu Province, China, 2020. Emerging Infectious Diseases. 2020; 26 (6).

34. Giovanetti M, Benvenuto D, Angeletti S, Ciccozzi M. The first two cases of 2019-nCoV in Italy: where they come from?. Journal of Medical Virology. 2020; 92 (5): 518-21.

35. Walter S. Japan confirms first case of infection from Wuhan coronavirus; Vietnam quarantines two tourists. The Straight Times; Published January 16, 2020.

36. Ministers Department of Health. First confirmed case of novel coronavirus in Australia; January 25, 2020.

37. Michelle LH, Chas DB, Scott L, Kathy HL, John W, Hollianne B, et.al, First case of 2019 novel coronavirus in the United States. The New England Journal of Medicine. 2020; 382: 929-36.

38. Wood F. Coronavirus: France confirms three cases of deadly virus. Independent; January 24, 2020.

39. Wright R. How Iran became a new epicenter of the coronavirus outbreak. The New Yorker; February 28, 2020.

40. Shahid A. Two coronavirus cases confirmed in Pakistan. Pakistan Today; February 27, 2020.

41. Daily Sabah. Turkey remains firm, calm as first coronavirus case confirmed; March 11, 2020.

42. Popo Maja. Minister Zweli Mkhize reports first case of Coronavirus Covid-19: first case of Covid-19 coronavirus reported in SA. South African Government: Department of Health; March 5, 2020.

43. World Health Organization. Coronavirus disease (COVID-19) advice for the public: Mythbuster; 2020.

44. World Health Organization. Coronavirus disease 2019 (COVID-19): situation report-71; 2020.

45. Wang C, Horby PW, Harden FG, Gao GF. A novel coronavirüs outbreak of global health concern. The Lancet. 2020; 395: 470-3.

46. Centres for Disease Control and Prevention. Coronavirus disease-19: how to protect yourself; 2020.

47. Centre for Disease Control and Prevention. CDC Newsroom: first travel-related case of 2019 novel coronavirus detected in United States; January 21, 2020.

48. Lu H. Drug treatment options for the 2019-new coronavirus (2019nCoV). Bioscience Trends. 2020; 14 (1): 69-71.

49. Cao B, Wang Y, Wen D, Liu W, Wang J, Fan G, et al. A Trial of lopinavir-ritonavir in adults hospitalized with severe Covid-19. The New England Journal of Medicine. 2020; 382: 1787-99.

50. Li G, De Clercq E. Therapeutic options for the 2019 novel coronavirus (2019-nCoV). Nature Reviews Drug Discovery. 2020; 19 (3): 149-50.

51. Yao X, Ye F, Zhang M, Cui C, Huang B, Niu P, et al. In vitro antiviral activity and projection of optimized dosing design of hydroxychloroquine for the treatment of severe acute respiratory syndrome coronavirus 2 (SARS-CoV-2). Clinical Infectious Diseases. 2020; 71 (15): 732-9.

52. Martinez MA. Compounds with therapeutic potential against novel respiratory 2019 coronavirus. Antimicrobial Agents and Chemotherapy. 2020; 64: e00399-20.

53. Bao L, Deng W, Huang B, Gao H, Liu J, Ren L, et al. The 
Oti et al, Traveler's Infection: Understanding SARS-CoV-2 as a Potential Agent

Pathogenicity of SARS-CoV-2 in hACE2 transgenic mice. bioRxiv.

2020;

54. Duddu P. Coronavirus outbreak: vaccines/drugs in the pipeline for Covid-19. Clinical trials arena. com; 2020 [Archived from the original on February 19, 2020].

55. NhuThao TT, Labroussaa F, Ebert N, V'kovski P, Stalder H, Portmann J, et al. Rapid reconstruction of SARS-CoV-2 using a synthetic genomics platform. Nature. 2020; 582: 561-5. 